challenging, multimodal approach with transdural ONYX embolization, transvenous embolization or even SRS can be considered. Further assessment of natural history of patients with near occlusion is warranted.

Disclosures T. Shigematsu: None. J. Fifi: None. A. Berenstein: None.

\section{0-026 CLEARANCE OF CHRONIC SUBDURAL HEMATOMAS FOLLOWING MIDDLE MENINGEAL ARTERY EMBOLIZATION}

J Catapano*, A Ducruet, K Rumalla, C Nguyen, V Srinivasan, C Rutledge, T Cole, J Baranoski, M Lawton, A Jadhav, F Albuquerque. Neurosurgery, BNI, Phoenix, AZ

\subsection{6/neurintsurg-2021-SNIS.26}

Background Chronic subdural hematomas (cSDHs) are one of the most common neurosurgical pathologies encountered. Recently, middle meningeal artery (MMA) embolization has emerged as an alternative to surgery with the potential for reduction in treatment failures. However, existing literature does not address the rates of $\mathrm{cSDH}$ resolution following MMA embolization nor variables associated with rapid clearance. The present study analyses clearance rates of cSDHs following MMA embolization.

Methods All patients who underwent a MMA embolization for a cSDH at a single center from $1 / 1 / 2018$ to $12 / 31 / 2020$ were retrospectively analyzed. Patients without sufficient follow-up scans of at least 20 days post-embolization were excluded. Patient characteristics, demographics, and technical details were examined. Outcomes analyzed included resolution and/or near complete resolution (defined as $<5 \mathrm{~mm}$ on axial head computed tomography) at 30-, 90-, and 180-days following embolization. An additional univariate analysis for factors associated with rapid clearance (defined as a rate of clearance $>0.5 \mathrm{~mm} /$ day) was performed. A subsequent stepwise multivariable logistic regression analysis for variables predictive of rapid clearance was performed for all factors with a p-value $<0.2$ on univariate analysis.

Results During the study period, 76 patients underwent a MMA embolization. 10 patients were excluded. In the 66 patients, there were a total of $84 \mathrm{cSDH}$ s treated. The mean hematoma size prior to embolization was $16.9 \mathrm{~mm}$ (sD 4.8) Both anterior and posterior branches were embolized in 61 (72\%) cSDHs and distal embolization was achieved in 57 (68\%) hemorrhages. There was one complication (1\%) reported (a CVA) and $3(4 \%)$ cSDHs required surgical rescue. There was a significant difference in mean $\mathrm{CSDH}$ size at 30 -

Abstract 0-026 Table 1 Characteristics of chronic subdural hematoma patients treated with middle meningeal artery embolization

\begin{tabular}{ll}
\hline Characteristic & $\mathbf{N}=66(\%)$ \\
\hline Age (yr), mean (SD) & $70 \pm 11$ \\
Male sex & $51(77)$ \\
GCS Presentation & $14 \pm 2$ \\
GCS Discharge & $14 \pm 2$ \\
Charlson Comorbidity Index & $4.7 \pm 2$ \\
Previous trauma & $42(64)$ \\
Preoperative Anticoagulant or Antiplatelet & $32(48)$ \\
Bilateral & $18(21)$ \\
\hline
\end{tabular}

Abstract 0-026 Table 2 Characteristics and outcomes of Chronic Subdural hematomas treated by middle meningeal artery embolization

\begin{tabular}{ll}
\hline Characteristic & $\mathbf{N}=84(\%)$ \\
\hline Hematoma Size (mm) & $16.9+/-4.8$ \\
& \\
Combined with Surgery & $31(37)$ \\
Radial* & $65(77)$ \\
Embosylate & \\
Onyx & $66(79)$ \\
Particles and/or coils & $13(16)$ \\
nBCA & $5(6)$ \\
Both Anterior and Posterior Branches Embolized & $61(72)$ \\
Distal Penetration & $57(68)$ \\
Complication & $1(1)$ \\
Surgical Rescue & $3(4)$ \\
\hline *One patient required crossover from radial to femoral due to inability to catheterize left internal carotid artery.
\end{tabular}

Abstract 0-026 Table 3 Average hematoma size $(\mathrm{mm})$ and percent of patients with complete and or near complete resolution at 30-, 90-, and 180-days post embolization

\begin{tabular}{lllll}
\hline & & & 180 Day (N=73) & P-Value \\
Yariable & 30 Day $(\mathrm{N}=84)$ & 90 Day $(\mathrm{N}=72)$ & & \\
\hline Size & $8.8 \pm 4.3$ & $3.4 \pm 3.0$ & $1 \pm 1.7$ & $<0.001$ \\
$\begin{array}{llll}\text { Complete/ Near Complete } \\
\text { Resolution }\end{array}$ & $15(16)$ & $45(63)$ & $67(92)$ & $<0.001$ \\
\hline
\end{tabular}

(8.8 mm, sD 4.3), 90- (3.4 mm, sD 3.0), and 180-days (1, sD 1.7) post-embolization $(\mathrm{p}<0.001)$. A greater percentage of cSDHs were found to have complete and/or near complete resolution at 180-days (92\%) compared to 90- (63\%) and 30days $(16 \%)$ post-embolization $(\mathrm{p}<0.001)$. On univariate analysis for factors associated with rapid clearance rates; bilateral embolization, female gender, distal penetration, and combined anterior and posterior embolization were associated with p-values $<0.2$. On stepwise multivariable logistic regression analysis, only distal penetration was found to be associated with rapid rates of clearance (OR 3.9, 95\% CI 1.4-11.1, p=0.011). Conclusion The majority of cSDHs undergoing MMA embolization will have complete and/or near complete resolution by 90-days post-procedure. Furthermore, distal MMA penetration may be associated with more rapid clearance of hematoma.

Disclosures J. Catapano: None. A. Ducruet: None. K. Rumalla: None. C. Nguyen: None. V. Srinivasan: None. C. Rutledge: None. T. Cole: None. J. Baranoski: None. M. Lawton: None. A. Jadhav: None. F. Albuquerque: None.

\section{0-027 EMERGENT VERSUS DELAYED CAROTID ARTERY STENTING FOLLOWING INTRACRANIAL THROMBECTOMY FOR ACUTE STROKE WITH TANDEM OCCLUSION}

${ }^{1} \mathrm{~A}$ Garg* ${ }^{1}{ }^{1} \mathrm{C}$ Zevallos, ${ }^{1} \mathrm{D}$ Quispe Orozco, ${ }^{1} \mathrm{~A}$ Mendez Ruiz, ${ }^{1} \mathrm{M}$ Farooqui, ${ }^{2} \mathrm{O}$ Zaidat, ${ }^{1} S$ Ortega. 'University of lowa Hospitals and Clinics, lowa City, IA; ${ }^{2}$ St. Vincent Medical Center, Toledo, $\mathrm{OH}$

10.1136/neurintsurg-2021-SNIS.27

Introduction At present, there is lack of consensus about the optimal timing of carotid artery stenting (CAS) following 
intracranial endovascular thrombectomy (EVT) for anterior circulation large vessel occlusions (LVO) presenting with tandem occlusion. We aimed to evaluate the safety of emergent CAS performed concurrently with EVT in this patient population.

Methods We utilized the Nationwide Readmissions Database 2016-2017 to identify patients admitted due to acute ischemic stroke who underwent anterior circulation EVT as well as CAS either on the same day or within 90 days of initial presentation. Survey design methods were used to generate national estimates. Univariate and multivariable logistic regression analyses were performed to evaluate clinical outcomes in patients who underwent emergent CAS (same day as EVT) in reference to those with delayed CAS (day 2-90).

Results We identified 1,601 hospitalizations meeting the study inclusion criteria (mean \pm SD age: $66.2 \pm 12.4$ years, female $29.5 \%)$. Of these, $1,392(87.0 \%)$ underwent emergent CAS and $209(13.0 \%)$ underwent delayed CAS. Baseline characteristics between the two groups were similar except patients with emergent CAS were less likely be on anti-thrombotic medications and to have previous history of stroke; and patients with delayed CAS had lower disease severity, were more likely to have received intravenous thrombolysis, to have private insurance and to be admitted at large, teaching hospitals. After adjusting for age, sex, and the differences in baseline characteristics, patients with emergent CAS had higher odds of in-hospital mortality [odds ratio (OR) 6.17, $95 \%$ confidence interval (CI) $1.36-27.92$, p 0.018], prolonged mechanical ventilation (OR 5.24, 95\% CI 1.46-18.80, p 0.011), and discharge disposition other than to home (OR 1.88, 95\% CI 1.01-3.49, p 0.047). Other outcomes including intracranial hemorrhage, gastrostomy tube placement, length of stay, and hospital charges did not differ between the two groups.

Conclusions Delayed CAS might be a safer approach for carotid revascularization for patients presenting with tandem anterior circulation lesions undergoing EVT.

Disclosures A. Garg: None. C. Zevallos: None. D. Quispe Orozco: None. A. Mendez Ruiz: None. M. Farooqui: None. O. Zaidat: None. S. Ortega: None.

\section{0-028 USING HIGH-FREQUENCY OPTICAL COHERENCE TOMOGRAPHY TO PREDICT ANEURYSM OCCLUSION IN A PRE-CLINICAL MODEL} ${ }^{1,2} \mathrm{R}$ King*, ${ }^{1,2} \mathrm{M}$ Shazeeb, ${ }^{1} \mathrm{~V}$ Anagnostakou, ${ }^{1} \mathrm{C}$ Raskett, ${ }^{3} \mathrm{~J}$ Arends, ${ }^{3} \mathrm{H}$ Dixit, ${ }^{1} \mathrm{~A}$ Puri,
${ }^{1} \mathrm{M}$ Gounis. ${ }^{2}$ Radiology, UMASS Medical School, Worcester, MA; ${ }^{2} \mathrm{Bme}$, WPI, Worcester,
MA; ${ }^{3}$ Stryker, Fremont, CA

\subsection{6/neurintsurg-2021-SNIS.28}

Background Flow diversion has shown high levels of treatment success at long term follow-up. However, short term healing rates tend to be much lower allowing for the possibility of rupture in the interim. With the development of neurovascular High Frequency Optical Coherence Tomography (HF-OCT), we hypothesize that the ability to measure the degree of communicating malapposition (CM) will allow for better prediction of early aneurysm healing.

Methods Fifty-two rabbits were implanted with a flow diverter over a previously created elastase induced aneurysm on the right subclavian artery. Five days prior to implant, all animals were administered with $10 \mathrm{mg} / \mathrm{kg}$ aspirin and clopidogrel, which continued for at least 30 days post-implant. After device implantation, HF-OCT imaging was acquired to assess neck apposition of the flow diverter. At either 90- or 180days post-implant, catheter based digital subtraction angiography (DSA) was acquired from the origin of the subclavian artery. Aneurysm healing was assessed on the 5-point Darsaut scale.

To leverage the increased resolution of HF-OCT, a semiautomated processing pipeline was developed that only required identification of the vessel lumen and flow diverter struts. The aneurysms' neck was automatically identified (figure $1 \mathrm{~A}, \mathrm{~B}$ blue) along with any $\mathrm{CM}$ that was greater than $50 \mu \mathrm{m}$ between the vessel wall and the flow diverter (figure 1 A, B green). This resulted in three measurements of interest: area of the neck, area of the annular CM, and volume between the flow diverter and the vessel wall. Area of the $\mathrm{CM}$ was normalized to the neck size to control for neck size differences and compared against the 5 progressive healing scores. Segmentation of HF-OCT images and assessment of DSA was done by independent reviewers.

Results Of 52 rabbits, 43 (83\%) had both adequate HF-OCT and device placement to be considered for evaluation. No differences were seen in the occlusion scores between 90- and 180-day follow-up ( $\mathrm{p}=0.67$ ), so the timepoints were combined for CM assessment. Rates of normalized CM were found to be significantly different across the groups ( $\mathrm{p}<$ 0.001 , ANOVA), and in a sub-group analysis, the rate of normalized CM was statistically lower in both the aneurysms scored as 3 and 4 ( $\mathrm{p}<0.01$, Tukey post-hoc) with all interactions shown in figure $1 \mathrm{C}$.

Conclusions HF-OCT demonstrates the ability to detect the degree of CM of flow diverters at implant. This measurement is strongly predictive of aneurysm healing at both 90- and 180-days post-implant.

Disclosures R. King: None. M. Shazeeb: None. V. Anagnostakou: None. C. Raskett: None. J. Arends: 5; C; Stryker. H. Dixit: 5; C; Stryker. A. Puri: 1; C; Stryker. 2; C; Stryker. M. Gounis: 1; C; Stryker. 2; C; Stryker.
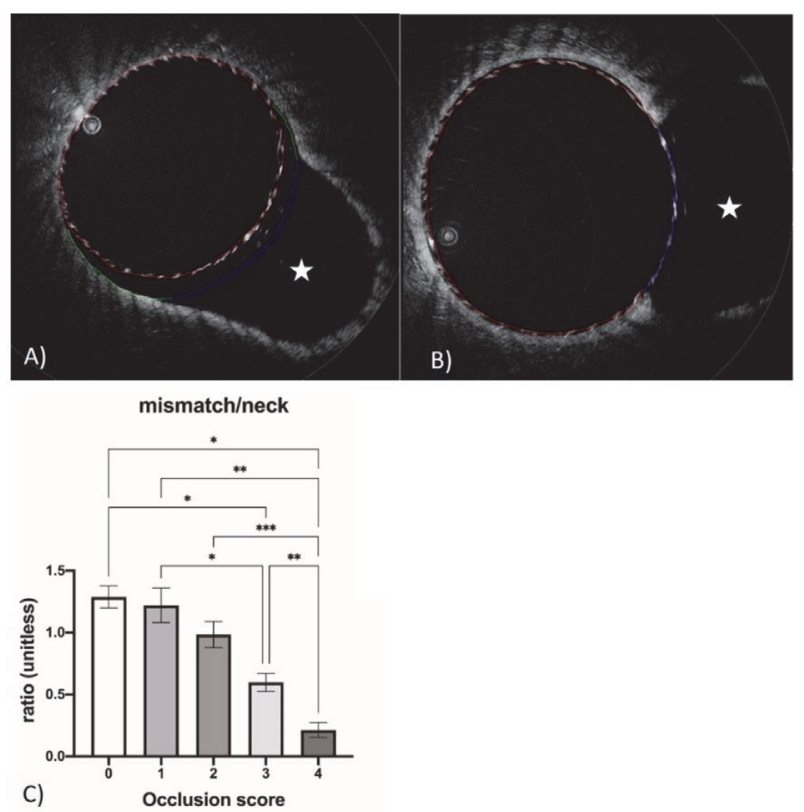

Abstract 0-028 Figure 1 